\title{
ACERCA DEL DINERO ELECTRÓNICO O DIGITAL
}

\author{
Juan José Blossiers Mazzini
}

Resumen

El presente artículo expone el alcance y dimensión del nuevo dinero electrónico, analizando su funcionamiento, procedimiento de emisión y garantías de seguridad al consumidor, abordando de manera detallada el escenario evolutivo de este sistema en el futuro. De esta manera, el autor fundamenta la importancia y repercusión del dinero electrónico en la economía, las finanzas así como en el comercio, finalizando con un interesante análisis del impacto de este nuevo medio de pago electrónico sobre el Derecho y su regulación internacional. En este sentido, el texto refiere las bondades de aplicar el dinero electrónico con la esperanza de que pronto sea de aplicación general.

\section{Summary}

The present article exposes the scope and dimension of the new electronic money, analyzing its opera!ion, emtssion procedure and security guarantees for the consumen explaining in detail the evotutionary scenery of this system in the future. In this way, the author fundaments the importance and repercuss ion of the electronic money in the Economy, the Finances as well as the Commerce, finahzing with an interesting analysis of the impact of this new electronic payment ahove the law and its international regulation. In that sense, the text refers the henefits of the use of the electronic money hoping soon to he of general application.

Sommaire

Dans le présent article, I 'autour expose la nouvelle dimensión de la monnaie électronique, en analysant leurs performances, des émissions et des garandes de sécurité pour te consommateur, abordant en détail le scénario évolutif de ce systéme dans lefutur. Ainsi, l'auteur fonde l'imporlance et Timpact de la monnaie électronique dans l'économie, des finances et le commerce. Finalement, iI fasse une intéressante analyse de I'impact de ce paiement électronique sur te Droit International et sa régulation. 


\section{INTRODUCCIÓN ${ }^{1 *}$}

Imagina usted ya no usar billetes y monedas sino simplemente tarjetas en donde esté representado el valor del dinero y poder efectuar compras y aceptación en los establecimientos que use.

El Dinero Electrónico o Digital viene a ser un sistema para adquirir créditos de dinero en cantidades relativamente reducidas. Este sistema consta de unidades o símbolos de valor monetario, debidamente cifrado que representa cantidades de dinero, que asumen forma digital; unidades que pueden ser convertidas en dinero físico. Este dinero electrónico se almacena en la computadora y se transmiten a través de redes electrónicas para ser gastado al hacer compras electrónicas a través de esa red de redes que es la Internet.

Teóricamente, el Dinero Electrónico o Digital podría utilizarse para cancelar compras por montos pequeños, hasta décimas de centavo de dólar o euro o sol y menos de ser el caso. Sin embargo, la mayoría de los comerciantes que aceptan dinero electrónico hasta el momento, lo emplean como una alternativa a otras formas de pago de adquisiciones de precio un tanto superior.

El Dinero Electrónico está pensado para realizar pagos por objetos de precio inferior a cinco (5) dólares, alternativa eficiente, ya que no se incurre en los costos que representan utilizar las tarjetas de crédito, sobre todo si hablamos de volúmenes monetarios pequeños.

El Dinero Electrónico funciona de la siguiente manera para un consumidor:

El primer paso es afiliarnos a un banco que ofrezca este sistema de Dinero Electrónico, luego debemos suscribir un contrato con alguna empresa proveedora del sistema, la cual nos proporcionará el software para instalarlo en la computadora. Este software permite bajar el dinero electrónico al disco duro de la computadora. La adquisición inicial de dinero se realiza contra nuestra cuenta bancaria o una tarjeta de crédito.

Una vez instalado el software en la computadora, procederemos a realizar nuestras compras en la red, asegurándonos que la tienda virtual que escojamos acepte Dinero Electrónico o Digital. Una vez escogido el producto y listos a realizar la compra, debemos simplemente hacer click en el botón de pago y el software de la tienda generará una solicitud de pago describiendo la mercancía, el precio, la fecha y la hora.

Una vez generada la solicitud y siempre que aceptemos, el software resta la cantidad del precio y crea un pago que es enviado al banco, verificado y luego depositado en la cuenta de la tienda virtual. Una vez que se ha concluido este proceso se notifica a la tienda virtual y esta envía la mercancía que hemos comprado.

Entre los sistemas de dinero electrónico o digital más usados en la actualidad tenemos el CyberCash, pariente de CyberCoin. E-cash y el sistema. DigiCash.

1 Premio Internacional de Interamerican Bar Association, Washington D. C.

Colaborador de la Revista de Derecho Bancario y Bolsa. España

Becario de la Suprema Corte de Buenos Aires -UIBA en el Diplomado de Derecho y Nuevas Tecnologías, Argentina

$\left(^{*}\right) \quad$ Dedicado a Sylvia 
La compañía Compaq está estudiando los resultados de su experiencia con el sistema MilliCent, sistema de dinero electrónico, recién inaugurado. De otro lado tenemos a IBM que se encuentra trabajando en un método de micropago llamado Minipay.

Actualmente, el dinero electrónico se enfrenta a algunas cuestiones que desalientan su uso, debido que para poner este sistema de pago en funcionamiento, los consumidores han de instalar en su computadora programas específicos; que representan un costo adicional a corto plazo. Asimismo, existen pocas tiendas virtuales que poseen estos programa con lo cual no se puede utilizar en toda la red; además de provocar una acumulación de pequeñas facturas que no es del agrado de gran cantidad de los consumidores.

Tal y como van las cosas, según Júpiter Communications, hacia el 2002 menos del $1 \%$ de las transacciones se realizarán con este sistema. A pesar de esto, los mayores revendedores aún apuestan por el Dinero Electrónico.

\section{JL Emisión de dinero electrónico}

La previsible irrupción de las operaciones comerciales a través de Internet se está perfilando como un nuevo canal que podría reportar grandes beneficios a las partes implicadas en la transacción, según se advierte en diferentes estudios e informes de las más prestigiosas consultoras a nivel mundial. A pesar de ello, existen todavía algunas barreras a superar para lo que ahora está en la menta de todos sea una realidad. En primer lugar, el aspecto relacionado con la seguridad, y en segundo, la necesidad de clarificar el concepto de dinero electrónico. Por ejemplo, el Banco Central Europeo - BCE en adelante- ha publicado un informe sobre dinero electrónico en el que analiza los riesgos asociados a un medio de pago que va a ser uno de los fundamentos del comercio electrónico dirigido al consumo y con el que se pretende solucionar, entre otros, el problema de los micropagos. El estudio se dirige a los sistemas de dinero electrónico basados en tarjetas de prepago o en software específico, desarrollado para realizar pagos a través de una red de telecomunicaciones como Internet. Entre las conclusiones del informe, cabe destacar las siguientes:

a. La emisión de dinero electrónico tendrá una importante incidencia en la política monetaria y obligará a asegurar la estabilidad de los precios y la función del dinero como unidad de cuenta.

b. Deberá analizarse la necesidad de desarrollar nuevas normas que garanticen: el funcionamiento eficaz de los sistemas de pago, la confidencialidad de las transacciones, la protección de los consumidores y de los comerciantes, la estabilidad de los mercados financieros, la protección frente a delitos informáticos.

c. También deberán establecerse los requisitos que deberá cumplir la emisión de dinero electrónico y en especial: la supervisión sometida a criterios de prudencia, normativa sólida y transparente, seguridad técnica que impida manipulaciones y falsificaciones, protección frente a delitos, especialmente el blanqueo de dinero. Así como el suministro de la información necesaria para generar estadísticas monetarias, garantía de conversión del dinero electrónico en moneda del banco central a reque- 
rimiento del poseedor del dinero electrónico, coeficiente de caja que obligue a los emisores de dinero electrónico a mantener unas reservas apropiadas.

d. Será necesario incrementar la cooperación entre las autoridades de supervisión de los países implicados para evaluar la integridad de los sistemas de dinero electrónico, en especial, en las operaciones transfronterizas.

e. Otro objetivo importante a perseguir es la interoperabilidad de los diferentes sistemas de dinero electrónico.

f. Finalmente, el BCE recomienda, inicialmente, limitar la emisión de dinero electrónico a las entidades de crédito, tal como las define el artículo $1^{\circ}$ de su primera Directiva sobre coordinación bancaria. Todo ello sin perjuicio de que en el futuro, dicha definición sea modificada para dar cabida a las entidades que emiten dinero electrónico y que no son entidades de crédito. El BCE aceptaría la existencia de un periodo transitorio durante el cual las entidades que actualmente están emitiendo dinero electrónico puedan seguir haciéndolo si cumplen los requisitos propuestos, con excepción del coeficiente de caja. Sistema de pagos del EURO en tiempo real. El Banco Central Europeo ha anunciado el proyecto TARGET, siglas de "Trans-European Automated Real-Time Gross Settlement Express Transfer". que será el sistema de pagos del euro en tiempo real. TARGET reúne a quince sistemas nacionales de pagos en tiempo real y el mecanismo de pagos del Banco Central Europeo que están interconectados para proporcionar una plataforma homogénea para el proceso de pagos transfronterizos. En circunstancias normales, los pagos llegarán a su destino en pocos segundos, después de ser adeudados en la cuenta del presentador. Todos los pagos recibirán el mismo tratamiento, independientemente de su importe. El acuse de recibo de cada orden de pago individual ejecutada correctamente será enviado al banco central nacional presentador en tiempo real. La liquidación será firme una vez que los fondos hayan sido abonados en cuenta. El dinero recibido será dinero abonado en cuentas de un banco central. Será posible reutilizar estos fondos varias veces al día. La liquidez quedará inmovilizada sólo el tiempo necesario para efectuar la liquidación en tiempo real. Para iniciar un pago transfronterizo en TARGET, los participantes transmitirán simplemente sus órdenes de pago al sistema de pagos del euro en tiempo real del que sean miembros (En el caso de España es SLBE), utilizando para ello el formato de mensaje nacional con el que están familiarizados y TARGET se ocupará del resto. El beneficiario recibirá el mensaje de pago en su formato nacional.

TARGET ha sido diseñado para cumplir tres objetivos principales:

a. Proporcionar un mecanismo seguro y fiable de liquidación bruta en tiempo real de los pagos transfronterizos; incrementar la eficacia de los pagos transfronterizos entre los países de la Unión Europea; y, sobre todo, responder a las necesidades de la política monetaria del Sistema Europeo de Bancos Centrales. 
b. La introducción del euro, permitirá a las entidades financieras y a las empresas con actividades internacionales en la Unión Europea centralizar sus operaciones de tesorería que están todavía distribuidas en diferentes monedas.

c. El uso de una sola moneda en vez de varias supondrá un ahorro considerable para todas las empresas que realizan actividades transfronterizas. Una condición previa para optimizar este ahorro es que los propios sistemas de pagos estén integrados. Todas las áreas de moneda nacional existentes tienen un sistema integrado de pagos del que deberá disponer también el área del euro. La instrumentación satisfactoria de la política monetaria única se reflejará en un tipo de interés uniforme en el mercado monetario. El mercado interbancario de la Unión Monetaria Europea (UME) requerirá, en primer lugar, que las entidades de crédito tengan tanto el incentivo como la capacidad de gestionar sus posiciones de liquidez de manera eficiente $y$, en segundo lugar, que las operaciones puedan ejecutarse fácilmente y rápidamente en toda el área del euro. A su vez, esto supone la existencia de un sistema integrado de pagos a fin de garantizar que la liquidez pueda transferirse de un participante a otro con seguridad, facilidad y rapidez dentro de la nueva área monetaria, tal como ocurre actualmente en las áreas monetarias nacionales.

El dinero electrónico también conocido como e-money, efectivo electrónico, moneda electrónica, dinero digital, efectivo digital o moneda digital, se refiere a dinero que se intercambia sólo de forma electrónica.

Típicamente, esto requiere la utilización de una red de ordenadores, la Internet y sistemas de valores digitalmente almacenados. Las transferencias electrónicas de fondos (TEF) y los depósitos directos son ejemplos de dinero electrónico. Asimismo, es un término colectivo para criptografía financiera y tecnologías que los permitan.

Si bien el dinero electrónico ha sido un interesante problema de criptografía véase por ejemplo el trabajo de David Chaunt y Markus Jakobsson-, hasta la fecha, el uso de dinero en efectivo digital se ha efectuado relativamente a baja escala. Uno de los pocos éxitos ha sido sistema de tarjeta Octopus en Hong Kong, que comenzó como un sistema de pago de tránsito masivo y se ha utilizado ampliamente como un sistema de dinero electrónico. Singapur también ha implementado un sistema de dinero electrónico para su sistema de transporte público (tren, autobús, etc), que es muy similar al de Hong Kong y la tarjeta Octopus basada en el mismo tipo de tarjeta (FeliCa). Otra aplicación exitosa se encuentra en los Países Bajos, conocida como Chipknip.

3. Sistemas alternativos

Técnicamente, el dinero electrónico o digital es una representación, o un sistema de débitos y créditos, destinado - pero no limitado a esto- al intercambio de valores en el marco de un sistema, o como un sistema independiente, pudiendo ser en línea o no. El término dinero electrónico también se utiliza para referirse al proveedor 
del mismo. Una divisa privada puede utilizar el oro para ofrecer una mayor seguridad, como la divisa de oro digital. Un sistema de divisas digital puede ser plenamente respaldado por el oro (como e-gold y c-gold), no respaldados en oro, o de ambos sistemas (como e-Bullion y Liberty Reserve). Además, algunas organizaciones privadas, como las Fuerzas Armadas de los Estados Unidos usan divisas privadas como el Eagle Cash.

Muchos de los sistemas electrónicos venden sus divisas directamente al usuario final, tales como Paypal y WebMoney. pero otros sistemas, tales como e-gold. venden sólo a través de terceros como las casas de cambio de moneda digital.

En el caso de la tarjeta Octopus en Hong Kong, se trabaja de manera similar a los depósitos bancarios. Después que tarjeta Octopus Limited recibe dinero en depósito de los usuarios, el dinero se deposita en bancos, lo cual es similar al método de las tarjetas de débito donde los bancos emisores redepositan el dinero a los bancos centrales.

Algunas divisas locales, como los sistemas de cambio local, trabajan con transacciones electrónicas. El Cyclos Software permite la creación electrónica de divisas locales. El sistema Ripple es un proyecto para desarrollar un sistema de distribución de dinero electrónico independiente de la moneda local.

\section{Dinero electrónico anónimo fuera de línea}

Con el dinero electrónico anónimo fuera de línea conocido como off-line el comerciante no tiene que interactuar con el banco antes de aceptar dinero por parte del usuario. En lugar de eso puede recoger múltiples monedas gastadas por los usuarios y depositarlas posteriormente en el banco. En principio esto se puede hacer fuera de línea, es decir, el comerciante podría ir al banco con sus medios de almacenamiento para intercambiar el efectivo electrónico por dinero en efectivo. No obstante, el comerciante debe asegurarse que el dinero electrónico del usuario, o bien será aceptado por el banco, o el banco será capaz de identificar y castigar a los usuarios que traten de engañar por esta vía. De esta forma, un usuario no tiene posibilidad de utilizar la misma moneda dos veces (doble gasto). Los sistemas de efectivo electrónico off-line también tienen la necesidad de protegerse contra los posibles engaños de los comerciantes, es decir, los comerciantes que deseen depositar una moneda dos veces (y luego culpar al usuario).

En criptografía el efectivo electrónico anónimo fue presentado por David Chaum. Solía hacer uso de firma digital ciega para lograr hacer imposible relacionar entre el retiro y transacciones de gastos. En criptografía, efectivo electrónico por lo general se refiere a dinero electrónico anónimo. Dependiendo de las propiedades de las operaciones de pago, se distingue entre efectivo electrónico en línea y fuera de línea (off-line). El primer sistema de efectivo electrónico fuera de línea fue propuesto por Chaum y Naor. Al igual que el primer sistema en línea, se basa en firma digital ciega RSA.

5. $\quad$ Evolución futura

Los ejes principales de desarrollo del efectivo digital son: 
La posibilidad de usarlo a través de una gama más amplia de hardware tal como tarjetas de crédito garantizadas,

Que las cuentas bancarias vinculadas, en general, se utilicen en un medio de Internet, para el intercambio con Micropagos seguros como en el sistema de las grandes corporaciones (PayPal).

Para el fomento de la evolución de la red en términos de la utilización de efectivo digital, una empresa llamada DigiCash está en el centro de atención con la creación de un sistema de efectivo electrónico que permite a los emisores vender moneda electrónica a algún valor. Cuando se adquieren vienen a nombre del comprador y se almacenan en su computadora o en su identidad en línea. En todo momento, el dinero electrónico se vincula a la empresa de efectivo electrónico, y todas las transacciones se realizan a través de esta, por lo que la compañía de efectivo electrónico asegura todo lo que se compra. Sólo la compañía tiene la información del comprador y dirige la compra a su ubicación.

Desarrollos teóricos en el ámbito de la descentralización del tradicional dinero centralizado están en marcha. Los sistemas de contabilidad que están apareciendo, tales como Altruistic Economics, son totalmente electrónicos, y puede ser más eficaces y más realistas por no asumir un modelo de transacción de Suma cero.

\section{6. $\quad$ Sistema de pasto electrónico}

Un sistema de pago electrónico realiza la transferencia del dinero entre comprador y vendedor en una compra-venta electrónica. Es, por ello, una pieza fundamental en el proceso de compra-venta dentro del comercio electrónico.

Como ejemplos de sistemas de pago electrónico nos encontramos las pasarelas de pago o TPV-virtual para el pago con tarjeta y los sistemas de monedero electrónico.

El comercio electrónico por Internet se ofrece como un nuevo canal de distribución sencillo, económico y con alcance mundial las 24 horas del día todos los días del año, y esto sin los gastos y limitaciones de una tienda clásica: personal, local, horario, infraestructura, etc. Los principales retos que presentan los EPS (sistemas de pago electrónicos) en Internet son los siguientes:

\section{1. ;Cómo funciona?}

En el pago con tarjeta, la pasarela de pago valida la tarjeta y organiza la transferencia del dinero de la cuenta del comprador a la cuenta del vendedor.

El monedero electrónico, sin embargo, almacena el dinero del comprador en un formato electrónico y lo transfiere al sistema durante el pago. El sistema de pago valida el dinero y organiza la transferencia a la cuenta del vendedor. También existe la posibilidad de que el sistema de pago transfiera el dinero electrónico al monedero electrónico del vendedor actuando en este caso como un intermediario entre ambos monederos electrónicos. 

compras:

Dentro de los posibles mercados de pago se distinguen tres modalidades de

Encontrar clientes y negocios que se arriesguen en un producto que está todavía en las primeras fases de introducción. Se trata del mayor reto y ha provocado un efecto de "quien es primero: la gallina o el huevo": los Bancos y las Compañías de Software no pueden obtener clientes sin vendedores, y a la inversa.

Garantizar la seguridad. Este es quizás el mayor problema para la gente interesada en realizar compras electrónicas. La mayor parte de la gente teme dar su número de tarjeta de crédito, número de teléfono o dirección porque no sabe si alguien será capaz de utilizar esa información sin su consentimiento. Es interesante comprobar que la mayoría de la gente no se lo piensa dos veces antes de comprar cosas por teléfono, pero les incomoda hacerlo a través de su PC. El estándar SET está alejando este miedo mediante un cifrado de los datos de la tarjeta, de forma que sólo el banco y el cliente puedan leerlos.

Garantizar el anonimato. Este es también un factor importante en algunos tipos de pago, en concreto en el pago mediante dinero electrónico. Sin embargo, gran parte de la gente se ha acomodado a las tarjetas de crédito y débito. El dinero electrónico anónimo tiene un mercado potencial, pero puede no ser tan grande como se espera.

Los sistemas de pago empleados en Internet pueden englobarse en cuatro categorías:

\subsubsection{Cajeros Electrónicos}

Se trata de sistemas en los cuales los clientes abren unas cuentas con todos sus datos en unas entidades de Internet. Estas entidades les proporcionan algún código alfanumérico asociado a su identidad que les permita comprar en los vendedores asociados a las entidades.

Dinero Electrónico (Anónimo e Identificado):

El concepto de dinero electrónico es amplio, y difícil de definir en un medio tan extenso como el de los medios de pago electrónicos (EPS). A todos los efectos se definirá el dinero electrónico como aquel dinero creado, cambiado y gastado de forma electrónica. Este dinero tiene un equivalente directo en el mundo real: la moneda. El dinero electrónico se usará para pequeños pagos (a lo sumo unos pocos miles de euros).

El dinero electrónico puede clasificarse en dos tipos:

a Dinero on-line:

Exige interactuar con el banco (vía módem o red) para llevar a cabo una transacción con una tercera parte. También existen monedas puramente electrónicas como e-gold. 


\section{b. Dinero offline:}

Se dispone del dinero en el propio ordenador, y puede gastarse cuando se desee, sin necesidad de contactar para ello con un banco. Estos sistemas de dinero electrónico permiten al cliente depositar dinero en una cuenta y luego usar ese dinero para comprar cosas en Internet.

\subsubsection{Cheques Electrónicos}

Los métodos para transferir cheques electrónicos a través de Internet no están tan desarrollados como otras formas de transferencia de fondos. Los cheques electrónicos podrían consistir algo tan simple como enviar un e-mail a un vendedor autorizándole a sacar dinero de la cuenta, con certificados y firmas digitales asociados. Un sistema de cheques puede ser considerado como un compromiso entre un sistema de tarjetas de crédito y uno de micropagos o dinero electrónico (anónimo).

\subsubsection{Tarjetas de Crédito}

Los sistemas de tarjetas de crédito en Internet funcionarán de forma muy similar a como lo hacen hoy en día. El cliente podrá usar si lo desea su tarjeta de crédito actua para comprar productos en una tienda virtual. La principal novedad consiste en el desarrollo del estándar de cifrado SET (Secure Electronic Transaction) por parte de las más importantes compañías de tarjetas de crédito.

La pregunta que se plantea en estos momentos es cual de los nuevos métodos de pago sobrevivirá al test del mercado. Resulta muy probable que el mayor ganador sea la tarjeta de crédito adaptada al comercio electrónico.

Se dice que el dinero no es más que información. Por tanto cualquier sistema de intercambio de información es susceptible de utilizarse para intercambiar dinero. Pero la Internet no se diseñó pensando en transacciones comerciales. Estas se realizaban, y se continúan realizando, a través de redes especiales (lo que los americanos suelen llamar Banknet). Los objetivos primordiales de la Internet eran facilitar la circulación de información científica y simplificar la interconexión de redes y equipos diversos, dejando un poco de lado los temas de seguridad. Ni siquiera la influencia militar, que había impulsado el proyecto en sus principios, ayudó a paliar esta deficiencia. El Departamento de Defensa de los Estados Unidos creó su propia red segura de comunicaciones y no consideró pertinente que información sensible circulara por la Internet. El resultado es que la Internet es un canal básicamente inseguro.

La entrada de empresas y servicios comerciales en la Internet atraídas por ventajas competitivas, por el prometedor segmento de mercado formado por los usuarios, o simplemente porque es el único prototipo existente de las llamadas "autopistas de la información" se ha ido progresivamente acelerando. Inicialmente muchas entidades se han limitado a una especie deposición de observación, de hacer notar su presencia en la red por motivos de imagen, esperando poder ampliar el espectro de operaciones posibles cuando consideren el tema maduro. Esta persistente implantación hizo que superaran por primera vez en nodos conectados a las instituciones edu- 
cativas y de investigación a finales del año 1994 (a principios de 1995 ya había más de 32.000 empresas conectadas en EEUU según datos de la Internet Society). El cambio de la Internet de una red primariamente dedicada a temas académicos hacia una red de propósitos generales (comercio, información, ocio, etc.) estaba servido.

De entre las causas que se alegan como justificación para el lento desarrollo del comercio electrónico, la más frecuente suele ser la falta de seguridad.

Esa imagen que transmiten los que desconocen la realidad de la red, de que Internet es una especie de submundo plagado de peligrosos hackers que se pasan día y noche esperando que introduzcamos el número de nuestra tarjeta de crédito para robarlo, es completamente falso.

Y lo más triste es que los mecanismos de seguridad existen, aunque parece que nadie está interesado en usarlos. Sistemas como el protocolo SET, desarrollado y operativo hace tiempo, no consigue hacerse un hueco en el mercado, a pesar de que tanto comerciantes como compradores se verían muy favorecidos por su utilización.

Pero no es de SET de quien vamos a hablar hoy, sino de del dinero electrónico, un medio de pago de quien podríamos decir que es el gran desconocido de la red, un sistema que tampoco acaba de encontrar su hueco en el comercio electrónico, a pesar de que, al igual que SET, cumple con todos los requisitos para ser considerado un medio completamente seguro, ostentando, además, las mismas funciones que el dinero físico, tanto monedas como billetes.

A diferencia de los medios de pago habitualmente utilizados en el comercio electrónico, tarjetas de débito y crédito, el dinero electrónico no depende directamente de mecanismos de pago existentes, como sería el funcionamiento de un TPV virtual o pasarela de pagos, sino que representa en sí mismo una nueva forma de pagar, un nuevo tipo de dinero, que, en determinadas condiciones, podría incluso ser utilizado en tiempo real sin necesidad de que una entidad financiera autorizase la transacción.

Para utilizar el dinero electrónico existen dos tecnologías distintas: los llamados "monederos electrónicos" y los "números firmados", que analizamos someramente a continuación:

\section{a. Monederos electrónicos}

Los Ilamados wallet en terminología anglosajona, y cuya traducción sería "billetero", son tarjetas que incorporan un chip, las llamadas tarjetas inteligentes o smart cards, donde se almacenan cantidades de dinero que previamente se han descontado de una cuenta bancaria.

El poseedor de la tarjeta dispone del dinero de forma anónima y puede gastarlo cuando y como quiera sin necesidad de conseguir la autorización de la entidad bancaria, ya que el dinero está en su monedero electrónico y no en el banco.

Este sistema ya se está utilizando para compras físicas en el mundo real, con las tarjetas monederas para compras de pequeño importe. Sin embargo, para usarlas en Internet requerirían el uso de unos lectores especiales que deberían incorporarse a los ordenadores, ya que el principal problema de estas tarjetas inteligentes es que 
deben tener una forma de comunicarse con el sistema para transferirle la información sobre el dinero de que dispone y la fecha de expiración para su uso.

Es evidente que la utilización de un lector acoplado al Computador Personal elimina la posibilidad de fraude, ya que el sistema exige la presencia física de la tarjeta, y no simplemente el conocimiento del número como pasa actualmente con las pasarelas de pago. Sin embargo, la necesidad de un terminal especial complica considerablemente la flexibilidad necesaria para que los usuarios puedan hacer compras desde donde quieran (no necesariamente desde su propio ordenador) y cuando lo deseen.

\section{b. Números firmados}

Este sistema de dinero electrónico se basa en que la entidad bancaria emite unos números aleatorios y los firma con su clave privada, quedando registrados en la base de datos de la entidad. El valor que tienen los números firmados depende de la longitud del número, y se pueden fraccionar cambiándolos en la propia entidad. Para decirlo de una forma comprensible, es como si el banco emitiera billetes numerados, aunque no tienen presencia física.

Los usuarios pueden pedirle al banco, a través de la red, los números que quieran, y la entidad se los suministra a cambio de un cargo en su cuenta o tarjeta, quedando el cliente, a partir de ese momento, habilitado para utilizar dichos números cuando y como desee.

Este medio de pago basado en números firmados no cuenta aún con difusión en la red, no siendo familiar ni para comerciantes ni para clientes. La causa es doble: de una parte, la complejidad técnica y la ausencia de estándares y regulaciones aceptadas por la banca, y de otra, la inexistencia de una legislación que proteja a los usuarios de los fraudes que pudieran sufrir al utilizar este procedimiento.

c. Esquema de funcionamiento del dinero electrónico

Es evidente que el dinero electrónico es un sistema análogo a la divisa extranjera en el sentido de que puede ser cambiado por dinero real en cualquier momento, para lo que se hace preciso que exista un banco central virtual, que es quien emite los números firmados.

El funcionamiento de este medio de pago es el siguiente:

El cliente tiene que abrir una cuenta on-line con el banco central virtual para cambiar su dinero tradicional por dinero electrónico.

El dinero electrónico que los clientes han comprado se guarda en un "monedero electrónico".

A partir de ese momento, los clientes pueden hacer uso del dinero electrónico con los comerciantes que lo acepten. 
Los comerciantes usan un software facilitado por el banco central virtual para aceptar el dinero electrónico, que posteriormente cambian por dinero tradicional en el banco central virtual.

La clave está en que cada moneda o billete electrónico tiene un número de serie, por lo que sólo puede ser emitido o cambiado una única vez por dinero real en el banco central virtual.

Las transacciones realizadas con dinero electrónico están protegidas por el sistema de criptografía de clave pública.

La intimidad de los clientes queda protegida al efectuarse el cambio del dinero electrónico por dinero tradicional, ya que el banco central virtual no revela su identidad. Es decir, al contrario de lo que sucede con las tarjetas de crédito o débito, en que la identidad del titular es conocida por el comerciante, el dinero electrónico es válido por sí mismo, sin que el comerciante necesite conocer quién es la persona que lo posee y lo está utilizando para hacer una compra.

¿Llegará el dinero electrónico a popularizarse convirtiéndose en una medio habitual de pago en la red? Es difícil saberlo. Sólo el tiempo nos lo dirá.

\section{7. $\quad$ ¿Oué dicen los expertos?}

En la Reunión del Centro Latinoamericano de Estudios Monetarios CEMLA en Bolivia llegaron a los siguientes problemas: problemas de política en el comercio electrónico.Con respecto al dinero electrónico:

Hasta el momento, la creación de dinero electrónico puede abordarse mediante los instrumentos jurídicos y reglamentarios establecidos.

En los últimos años, el dinero electrónico (es decir, las unidades o los signos dotados de valor monetario que tienen forma digital y se transmiten a través de las redes electrónicas) se ha convertido en un medio de pago «en línea». Esto ha suscitado algunas inquietudes en relación con la protección de los consumidores, el cumplimiento de la ley, la supervisión y reglamentación de los emisores de dinero electrónico y los efectos en la política monetaria. Dada la importancia de un sistema financiero estable y unas políticas monetarias adecuadas para la estabilidad macroeconómica, el Banco de Pagos Internacionales ha realizado una evaluación inicial detallada de estas cuestiones, en la que se basa en gran medida el examen que figura a continuación (Banco de Pagos Internacionales, 1997). El Banco hace referencia a varias consideraciones fundamentales que quizá los consumidores, los proveedores y las autoridades deseen tener en cuenta al aplicar y utilizar productos monetarios electrónicos y al elaborar políticas nacionales. Debe señalarse, no obstante, que, hasta el momento, las expectativas en relación con la creciente importancia del dinero electrónico no se han materializado por completo. Se ha descubierto que al público no le gusta pagar por el uso de ese medio en las transacciones muy pequeñas, medidas por contador, para las que se concibió originalmente el dinero electrónico. Además, las cantidades mínimas para el pago mediante tarjetas de crédito (la principal alternativa al dinero electrónico) siguen disminuyendo.

Por otra parte, actualmente se está intentando eliminar las inquietudes en materia de seguridad que despiertan los pagos en línea mediante tarjetas de crédito. 
La protección de los consumidores contra el fraude, la pérdida o la insolvencia de una institución que emita dinero electrónico no plantea muchas cuestiones distintas de las que surgen en el contexto de los medios de pago existentes, y los gobiernos pueden abordar esos problemas mediante las leyes y reglamentos vigentes. En cuanto al cumplimiento de la ley, en la actualidad, el dinero electrónico se utiliza sobre todo para transacciones de poco valor, que no suscitan, por el momento, especiales preocupaciones en relación con el blanqueo de capitales, la evasión fiscal o cualquier otro delito financiero. Sin embargo, si pueden transferirse grandes sumas en forma de dinero electrónico y si la capacidad de rastreo y los registros electrónicos siguen siendo insatisfactorios, las transferencias transfronterizas de dinero electrónico a países con disposiciones poco eficaces contra el blanqueo de capitales pueden constituir una opción atractiva para los delincuentes. A medida que evoluciona el dinero electrónico, se debe vigilar su potencial delictivo, y quizás sea necesario considerar la posibilidad de adoptar con el tiempo nuevas leyes y reglamentos.

Una tercera cuestión importante se refiere al efecto de la emisión de dinero electrónico en la reglamentación y supervisión de las instituciones financieras. Si las instituciones crediticias existentes emiten dinero electrónico, probablemente el marco reglamentario vigente baste para garantizar que se adopten precauciones adecuadas contra los riesgos opcracionales y en materia de liquidez y para mantener controles internos suficientes. Los demás emisores de dinero electrónico pueden necesitar un marco reglamentario específicamente adaptado a ellos pero, mientras sólo se trate de pequeñas sumas, quizá sea apropiado un régimen de supervisión más indulgente. Los pagos transfronterizos. en particular, pueden crear el temor de que los emisores se constituyan en sociedad o establezcan instalaciones en los países cuyas prescripciones reglamentarias sean menos rigurosas. Pese a estas preocupaciones, los pagos transfronterizos no deben verse innecesariamente obstaculizados por políticas nacionales excesivamente restrictivas.

Por último, la emisión de dinero electrónico podría afectar a los ingresos por concepto de ntonedaje y a la formulación de políticas monetarias de los bancos centrales. Es probable que el dinero electrónico reemplace al dinero en metálico, lo cual haría disminuir los ingresos de los bancos centrales por concepto de ntonedaje. Sin embargo, la reducción de esos ingresos sólo influirá en la financiación de las operaciones de los bancos centrales ( $y$ en la transferencia del residuo al presupuesto del Estado) si el dinero electrónico reemplaza un gran volumen de dinero en metálico. La formulación de la política monetaria podría verse afectada debido a efectos sobre la oferta y la demanda de dinero y sobre la velocidad de circulación de éste. No obstante, como no se prevé que, en un futuro próximo, haya una importante sustitución de dinero en metálico y depósitos por dinero electrónico, las repercusiones deberían ser insignificantes.

\section{Definición de Banca Electrónica v Dinero Electrónico}

La banca electrónica se refiere al suministro de productos y servicios bancarios para consumidores por medio de canales electrónicos

Estos productos y servicios pueden incluir la recepción de depósitos, préstamos, manejo de cuentas, asesoría financiera, pago electrónico de facturas y el sumi - 
nistro de otros productos y servicios de pago electrónico, como ser, dinero electrónico. Dos de los aspectos fundamentales de la banca electrónica son: las características de los canales de entrega y los medios disponibles a los consumidores para acceder a estos canales. Los canales de entrega más comunes incluyen redes "cerradas" y "abiertas". Las "redes cerradas" limitan el acceso a los participantes (instituciones financieras, usuarios, comerciantes y suministradores de servicios para terceros) que son miembros en virtud de un acuerdo específico.

Las "redes abiertas" no tienen estos requisitos de membresía. Actualmente, los productos y servicios de la banca electrónica son suministrados a los consumidores por medio de una variedad de dispositivos de acceso, como ser, terminales en los puntos de venta, cajeros automáticos, teléfonos, computadoras personales, smart cards y otros.

El dinero electrónico se refiere a un valor almacenado o mecanismos pagados por adelantado para la ejecución de pagos por medio de terminales en el punto de venta, transferencias directas entre dos dispositivos, o mediante redes abiertas de computación, como ser el Internet. Los productos de valor almacenado, incluyen mecanismos de hardware y "basados en tarjetas" (también llamados "monederos electrónicos") y mecanismos software o "basados en redes" (también llamados"efectivo digital"). Las tarjetas de valor almacenado pueden ser "uni propósito" o"multi-propósito". Las tarjetas de uni-propósito (por ejemplo, tarjetas para teléfonos) se usan para comprar un sólo tipo de bien o servicios, o productos de un sólo vendedor. Las tarjetas multi-propósito pueden ser utilizadas para una variedad de compras a varios vendedores.

Los bancos pueden participar en los esquemas de dinero electrónico como emisores, pero pueden también realizar otras funciones. Estas incluyen la distribución de dinero electrónico emitido por otras entidades, el rescate de las ganancias de las transacciones en dinero electrónico para los comerciantes, el manejo del procesamiento, compensación y liquidación de las transacciones de dinero electrónico y el manejo de registros de transacciones.

En nuestra realidad, el dinero electrónico comprende el valor monetario almacenado en un dispositivo electrónico con la finalidad de ser utilizado para la compra de bienes o servicio

\section{IL EL PAGO MEDIANTE DINERO ELECTRÓNICO}

\subsection{Marco conceptual}

\subsubsection{Consideraciones Previas}

Hablar de dinero electrónico es un tanto complicado y algo confuso, si tenemos en cuenta que, dinero electrónico es cualquier modalidad de pago que utilice tecnologías electrónicas, como por ejemplo las tarjetas.

Antes de proceder a definir el dinero electrónico, es necesario considerar dos aspectos fundamentales: el funcionamiento operativo de este medio de pago y las 
distintas modalidades que pueden ubicarse dentro de la denominación general "dinero electrónico".

Al abordar la primera de las cuestiones indicadas, podemos observar que el dinero electrónico se configura como una modalidad de las ya conocidas transferencias electrónicas de fondos, en el sentido que su uso implica un movimiento de fondos de una cuenta bancaria a otra con el objeto de realizar un pago a través de un medio electrónico.

En el caso del dinero electrónico nos ubicamos dentro de la categoría de las transferencias de crédito ya que es el comprador quien pone en marcha la operación correspondiente al girar instrucciones a su banco para que ejecute un pago.

El segundo de los aspectos planteados, nos conduce a una doble vertiente conceptual, la variedad de medios de pago existentes ubicados todos bajo la categoría general "dinero electrónico", obliga a hacer una diferenciación, en este sentido distinguimos el dinero electrónico en sentido amplio y el dinero electrónico en sentido restringido.

\subsubsection{Dinero electrónico en sentido amplio $v$ en sentido estricto}

En la primera de las orientaciones indicadas anteriormente, (dinero electrónico en sentido amplio), la noción "dinero electrónico" se identifica con cualquier sistema de pago que requiera para su funcionamiento una tecnología electrónica, abarcando esta denominación, las tarjetas electrónicas, los títulos valores electrónicos (cheques y letras de cambio), las cartas de crédito electrónicas, el dinero efectivo electrónico así como cualquier otra forma de pago que implique la existencia de un medio electrónico para hacerse efectivo.

En sentido restringido, la noción "dinero electrónico" alude al "dinero efectivo electrónico" o dinero digital (en atención a la tecnología actualmente utilizada), utilizándose esta expresión únicamente para referirse a las monedas y billetes electrónicos como sustitutos del dinero metálico o del papel moneda tradicionalmente conocido.

\subsubsection{Definición}

Según las consideraciones expuestas, podemos definir en forma general el dinero electrónico como un instrumento basado en el funcionamiento de una TEF, Transferencia Electrónica de Fondos, que tiene por objeto facilitar el pago en operaciones generalmente concertadas a través de redes de comunicación pudiendo asumir distintas formas según la voluntad de las partes negociantes.

Decimos que se trata de operaciones generalmente concertadas a través de redes de comunicación pues hay algunos casos, como las tradicionales tarjetas, que aun cuando implican el uso de medios electrónicos pueden ser utilizadas en cualquier establecimiento físico independientemente de la existencia de una red de comunicación. 
De otra parte hablamos del elemento volitivo como requisito indispensable para el uso del dinero electrónico, en el entendido que su aceptación siempre estará supeditada a la voluntad de las partes ya que no puede obligarse a nadie a recibir ni a efectuar un pago distinto del dinero efectivo tradicional calificado como de curso legal en cada una de las legislaciones existentes; así como los cheques y las tarjetas tradicionales requieren para su aceptación la manifestación del concurso de voluntades entre el vendedor y el comprador -y en algunos casos hasta del emisor del medio de pago-, de igual manera sucede con el dinero electrónico en cualquiera de sus distintas modalidades.

\subsection{Regulación jurídica internacional}

El concepto de medios electrónicos de pago lo encontramos en el ámbito europeo en la Recomendación 97/489/CE1 relativa a las transacciones efectuadas mediante instrumentos electrónicos de pago, en particular las relaciones entre emisores y titulares de tales instrumentos, que entiende por tales

"(...) aquellos que permiten efectuar transferencias de fondos, retirar dinero en efectivo y la carga de un instrumento de dinero electrónico en los cajeros automáticos, y el acceso a los fondos de la cuenta en una entidad (instrumentos de pago de acceso a distancia) (...)".

Al configurar una modalidad de las TEF, el dinero electrónico es regulado en forma general por la normativa que regula las TEF. No obstante esta regulación de carácter general, la preocupación por regular el dinero electrónico propiamente dicho, se ha hecho sentir sobre todo en el ámbito comunitario, donde existen diversos textos legales reguladores.

En materia específica de dinero efectivo electrónico, es de destacar la adopción de la Directiva 2000/46/CE del Parlamento Europeo y del Consejo, de 18 de septiembre del 2000, sobre el acceso a la actividad de las entidades de dinero electrónico y su ejercicio así como la supervisión cautelar de dichas entidades, (conocida comúnmente corno Directiva sobre entidades de dinero electrónico), como único texto legal sistematizado orientado a regular la emisión del dinero efectivo electrónico. La normativa contenida en la Directiva citada, establece un marco reglamentario orientado hacia el reconocimiento mutuo de la autorización y supervisión cautelar de las entidades emisoras de dinero electrónico que operan dentro del territorio comunitario, a través del establecimiento de una licencia única con supervisión en el Estado de origen, de modo que permita aprovechar las ventajas derivadas de la utilización del dinero electrónico y fomentar su uso.

En Estados Unidos las TEF son reguladas por primera vez en la EFTA sancionada por el Congreso el 10 de noviembre de 1978 e implementada a través de un reglamento especifico denominado "Regulation $-E$ " de la Federal Reserve Borrad

\subsection{Distintas categorías}

Si aludimos a la noción amplia del dinero electrónico, nos encontramos las siguientes categorías: 


\subsubsection{Las tarjetas electrónicas}

Dentro de la amplia clasificación de las tarjetas, nos referimos en esta oportunidad a las nuevas tarjetas surgidas como consecuencia del desarrollo tecnológico, esto es a los monederos electrónicos y a las tarjetas diseñadas en forma específica para realizar pagos en Internet, comúnmente conocidas como tarjetas virtuales.

\section{a. Los monederos electrónicos}

Los monederos electrónicos son tarjetas de prepago que permiten almacenar unidades monetarias en un microchip con la finalidad de realizar pequeñas compras, (cabinas telefónicas, taxis, kioscos, bares, pequeños comercios de alimentación) donde hasta ahora no se aceptaba la tradicional tarjeta de crédito y el consumidor debía realizar los pagos en efectivo.

El funcionamiento de los monederos electrónicos implica el almacenamiento previo a su uso- de una determinada cantidad de dinero a voluntad del titular de la tarjeta mediante una transferencia de la cuenta bancaria del usuario del monedero.

Con el dinero contenido en el monedero electrónico, el titular puede adquirir los productos y servicios de los proveedores adheridos al sistema, el uso de esta tarjeta implica su introducción en el dispositivo adecuado del proveedor de bienes y/o servicios a objeto de registrar la operación realizada y efectuar la correspondiente deducción del saldo, situación que en principio limita el uso de la tarjeta monedero en operaciones en Internet, no obstante su uso se está implantando a través de la instalación de un hardware específico en las computadoras de los usuarios que permite la lectura de las tarjetas.

El importe de las operaciones se registra tanto en la tarjeta a través de microchip, como en los registros informáticos del banco. La utilización del monedero electrónico, comporta para el titular la asunción de los riesgos en caso de utilización fraudulenta o irregular así como por su pérdida, incluso después de haberlo notificado al banco.

Entre los principales sistemas de pago que utilizan el monedero electrónico cabe mencionar el Sistema de Europay, MasterdCard y Visa (EMV), el European Electronic Purse (EEP), iniciativa del European Committee for Banking Standards (ECBS), el Conditional Access for Europe (CAFE) el sistema MONDEX y el Cyber- Cash5 . una de las tarjetas monedero que lleva más tiempo en el mercado es la tarjeta creada por la empresa Mondex, subsidiaria de MasterCard, esta tarjeta permite almacenar distintas divisas y contiene un programa de seguridad para proteger el dinero contenido en el monedero electrónico.

\section{b. Las tarjetas virtuales}

Las tarjetas para pagos en Internet representan la última novedad en materia de tarjetas electrónicas. Aún cuando para el pago en el comercio electrónico en Internet no es necesaria la configuración de una tarjeta especial en el sentido que po- 
demos pagar con las tradicionales tarjetas de crédito, débito o compra, las numerosas reclamaciones de los clientes por el uso fraudulento de las tarjetas, impulsaron a las diferentes instituciones financieras y propietarias de las marcas que emiten las tarjetas a trabajar en el diseño de tarjetas especiales para su uso específico en Internet.

En España, Banesto ha sido la institución pionera en lanzar al mercado la denominada Virtual Cash, se trata de un monedero electrónico de uso exclusivo para Internet, diseñado para efectuar pequeños pagos, la tarjeta no incorpora microchips, ni bandas magnéticas, sólo un número que sirve para cargarla en el cajero, tampoco está vinculada a una cuenta corriente y se puede solicitar on line, en la página Web de Banesto, su funcionamiento se basa en un número asignado a la tarjeta y la clave secreta que se utiliza para el recargo con la tarjeta de crédito o débito del cliente, y es aceptada en los establecimientos afiliados al sistema VirtualCash.

En Italia se está implantando el sistema de pago mediante lectores de tarjetas conectadas al PC del comprador. Se trata de un sistema que permite hacer los pagos en Internet sin enviar el número de la tarjeta de manera que ningún hacker pueda apropiarse de los datos del instrumento de pago ni descifrar los algoritmos matemáticos de cifrado de la información. El sistema es proporcionado por la empresa Mover, especializada en diseños de sistemas de seguridad para pagos electrónicos. Mediante un acuerdo contractual que incluye el pago correspondiente al servicio, la empresa expide directamente al usuario un kit compuesto de una tarjeta inteligente denominada Movercard y un lector para conectar al PC.

La firma electrónica del usuario está contenida en el microprocesador de la tarjeta de modo es posible reconocer su identidad, una vez insertada la tarjeta en el lector se teclea el PIN y se autoriza el pago, con este sistema es posible hacer compras en los establecimientos afiliados al sistema Mover.

\section{c. Sistemas de seguridad en los pagos con tarjetas}

El masivo uso de las tarjetas en Internet y la sensación de inseguridad en el usuario al transmitir sus datos a través de una red abierta, provocó gran preocupación en las empresas emisoras y gestoras de las tarjetas creando distintos sistemas de seguridad a objeto de solucionar estos problemas y fomentar su uso, uno de los protocolos creado específicamente para realizar estos pagos es el protocolo SET, diseñado por Visa y Masterd Card en conjunto con las grandes empresas informáticas.

El protocolo SET funciona mediante el cifrado del mensaje, el uso de la firma electrónica y los certificados digitales, todas las partes intervinientes en el proceso deben estar certificadas, lo cual implica la intervención de un prestador de servicios de certificación. En el pago mediante tarjetas con el uso del protocolo SET, además de la entidad emisora, la entidad negociadora o adquirente, el titular de la tarjeta y el comerciante, interviene una parte adicional denominada la "pasarela de pagos" (Gateway) se trata de una institución financiera que proporciona soporte a los comerciantes, cuya función consiste en procesar los pagos actuando como intermediario entre los diferentes bancos que participan en la transacción y el vendedor. En el sistema operativo, una vez que el comerciante recibe los datos de la tarjeta los envía directamente a la pasarela de pagos, (que también debe estar certificada), con la finalidad de obtener la correspondiente autorización o rechazo de la transacción. 
Para comprar con este protocolo se requiere un software SET, que es suministrado generalmente por la entidad emisora de la tarjeta, un certificado digital SET y un monedero digital. El certificado digital SET es emitido por la misma entidad emisora de la tarjeta y asegura la legitimidad en el uso de la misma, si se tiene más de una tarjeta electrónica, se requiere un certificado distinto para cada una; igualmente el vendedor necesitará un certificado digital diferente para cada marca de tarjeta que quiera aceptar, el uso de estos certificados proporcionan al comprador la misma seguridad que cuando paga con tarjeta en el establecimiento físico. El monedero digital denominado Wallet, funciona en sentido similar a una cartera física almacenando las diferentes tarjetas electrónicas que posee el comprador y su identificación personal.

La seguridad en la transacción en el pago con tarjetas mediante el uso del protocolo SET, se basa en el sistema de firma doble, el titular firmará el pedido con la firma pública del comerciante (pudiendo ser éste solamente quien descifre el mensaje con su clave privada) mientras que la parte referente al pago vendría cifrada con la clave pública de la entidad emisora o directamente con la clave pública de la pasarela de pagos según los casos, siendo éstos los únicos que podrán descifrar esta parte del mensaje mediante la aplicación de su clave privada.

Una vez que el mensaje ha sido cifrado, el comprador envía el pedido, el comerciante lo recibe y comprueba su veracidad, remite la orden a la pasarela de pagos, quien no tendrá acceso al contenido del pedido pero sí a los datos bancarios necesarios para autorizar o denegar la operación, ésta a su vez solicita la respuesta de la autorización a la respectiva entidad financiera (banco emisor o banco adquirente), enviando la respuesta al comerciante.

De esta manera se dice que la seguridad en la transacción es absoluta ya que mediante el sistema de certificados se garantiza la autenticidad de las partes mientras que el uso de la firma electrónica aporta las garantías de integridad y "० repudio mensaje.

No obstante la seguridad que aporta el uso del protocolo SET, su implantación ha sido criticada en el entendido de que se trata de un proceso complejo y lento al requerir sistemas asimétricos de cifrado y la comprobación de los distintos certificados de las partes, de manera que en cada comunicación, el receptor debe comprobar el origen de la transmisión por medio del sistema de jerarquías de certificados de los distintos proveedores que los hayan emitido.

De otro lado, los clientes deben darse previamente de alta en el sistema, solicitando la instalación del Wallet y la conexión con el proveedor de servicios de certificación para la correspondiente emisión de las claves, lo cual conlleva plazos entre una y dos semanas.

Debido a la complejidad del sistema SET, en la actualidad el protocolo de seguridad más utilizado es el SSL. EL protocolo SSL desarrollado por Netscape Communications Corporation, integrado al navegador, utiliza conjuntamente cifrado simétrico y asimétrico, encripta los números de la tarjeta proporcionando cifrado de datos, autenticación de servidores, integridad del mensaje y opcionalmente la autenticación del cliente, pero no consigue la confidencialidad, y no repudio del mensaje, sólo conseguida por el momento por el protocolo SET. SSL proporciona servicios de seguridad cifrando los datos intercambiados entre el servidor y el cliente mediante un 
algoritmo de cifrado simétrico. A diferencia del protocolo SET, el sistema SSL carece de capacidad para verificar la validez del número de tarjeta, autorizar la transacción y procesar la operación con el banco adquirente; la entidad emisora sólo asegura que mientras viajan los datos desde el navegador hasta el servidor no serán modificados, pero una vez que lleguen allí, los datos pueden ser manipulados.

\section{d. Cheques electrónicos}

En materia de cheques electrónicos susceptibles de uso en Internet, existen en la actualidad dos proyectos oficiales: el proyecto FSTC (Financial Services Techology Consortium) en Estados Unidos y el Proyecto MANDATE (Managing and Administrating Negotiable Documents and Trading them Electronically) en Europa. Al lado de estos proyectos existen diversas iniciativas de las grandes empresas y compañías informáticas para facilitar el pago de las compras a través de Internet en los que cabe resaltar el uso de Net Check, el Cashier Check y el Check Free.

En líneas generales, el funcionamiento de los citados proyectos se basa en la sustitución del soporte tradicional del cheque -el papel- por un soporte electrónico que permite emitir y enviar el cheque al tenedor a través de la Red; de igual forma, el tradicional talonario de cheques se sustituye por una chequera electrónica de bolsillo contenida en una tarjeta electrónica. La seguridad de estos sistemas se basa en el uso de algoritmos criptográficos que permiten garantizar la integridad de los datos contenidos en el cheque, evitar las duplicaciones y mantener oculto el número de cuenta del librador. El uso del cheque electrónico debe ser pactado con el banco o entidad financiera y debe insertarse dentro del contrato de cuenta corriente de igual forma como se pacta el uso del cheque tradicional ya que en virtud de este contrato es que la entidad se obliga a satisfacer al tenedor el importe del cheque. Debe existir una cláusula en el contrato que permita la emisión de cheques mediante el uso de la firma electrónica registrando los datos relativos a la firma del librador tal como se hace en los casos de las firmas manuscritas, para lo cual será necesario la intervención de una tercera parte, el prestador de servicios de certificación.

\subsection{Efectos de certificar los datos relativos a la firma electrónica del librador}

El sistema del E-check permite la emisión de cheques certificados mediante la certificación bancaria del instrumento, este procedimiento se lleva a cabo a través de la inserción de parte de la institución financiera de un sello de garantía avalado con su firma electrónica, garantizando al portador del cheque la existencia de una determinada disponibilidad a su favor, de esta manera funciona el sistema "Cashier Check", utilizado con mayor difusión en Estados Unidos.

\subsection{El caso específico del dinero electrónico}

El uso del dinero efectivo electrónico ha surgido recientemente como una alternativa a los pagos realizados con tarjetas ya que muchos usuarios de Internet se 
abstienen de realizar sus compras con tarjetas por la facilidad de rastrear la compra, sus nombres, números de tarjetas, gustos, preferencias etc. el dinero electrónico aporta un elemento adicional de seguridad, al no dejar rastros de la compra, ya que en principio es anónimo. La Directiva sobre regulación de las entidades emisoras de dinero electrónico define el dinero electrónico como un valor monetario representado por un crédito exigióle a su emisor, almacenado en un soporte electrónico, -por ejemplo, una tarjeta inteligente o el disco duro de una computadora- aceptado como medio de pago por empresas distintas de la entidad emisora, generado como un sustituto electrónico de monedas y billetes con la finalidad de realizar pagos de escasa cuantía por medios electrónicos. La definición citada abarca tanto al dinero efectivo contenido en los monederos electrónicos como el dinero generado a través de un software.

La Comunicación de la Comisión al Consejo, al Parlamento Europeo y al Comité Económico y Social sobre un Marco de actuación para la lucha contra el fraude y la falsificación de los medios de pago distintos del efectivo, se refiere a los "instrumentos electrónicos con valor almacenado" incluyendo en esta categoría las tarjetas prepagadas y dinero electrónico basado en un programa informático.

De los textos legales citados podemos diferenciar claramente dos tipos de dinero efectivo electrónico, el dinero contenido en un dispositivo electrónico -una tarjeta, en este caso nos referimos a los monederos electrónicos descritos anteriormente y el dinero efectivo generado a través de un programa de ordenador.

\subsection{El dinero electrónico generado a través de un prourama de computadora}

Para la generación del dinero efectivo electrónico a través de un programa de ordenadores necesario, en primer lugar, tener una cuenta bancaria en una institución financiera ya que son los fondos de esa cuenta los que permiten a su titular emitir dinero electrónico, en este sentido podemos decir que el dinero efectivo electrónico funciona de forma similar al cheque que es librado contra una disponibilidad de fondos.

Con el software apropiado se puede transferir dinero de la propia cuenta bancaria a través de Internet al disco duro del ordenador. En los billetes electrónicos, el papel moneda se sustituye por un conjunto de bits representativos de un determinado valor denominados tokens. que se almacenan en ficheros del disco duro del ordenador y que pueden transferirse a través de la Red a cambio de un producto o servicio. Para tener validez, los tokens deben ser similares al dinero metálico o de papel, así como en los billetes y monedas tradicionales el banco se compromete a su reconocimiento por un valor determinado, de la misma manera los billetes y monedas electrónicas deben estar respaldados por un determinado valor, tratándose en este caso de un valor prepagado, siendo la institución financiera la encargada de autenticar su emisión. Por otra parte deben reunir las mismas características de identificación de sus homólogos en papel:
a. estar identificados con un número de serie único,
b. contener el valor nominal,
c. estar fechados y
d. firmados por la entidad emisora. 
Para certificar su valor, el banco emisor firma los tokens con su firma digital y carga en la cuenta del usuario la cantidad de dinero real correspondiente al dinero digital generado.

Uno de los principales problemas que se presentan con el uso de este sistema es la facilidad de copiar el billete emitido electrónicamente y la posibilidad de cobrar el mismo billete más de una vez. Estos problemas se solucionan en principio, con la ayuda de técnicas criptográficas en la generación del dinero electrónico y con un control exhaustivo de parte de la institución financiera que respalda la emisión del dinero electrónico.

\subsection{Procedimiento de emisión}

Uno de los sistemas de dinero efectivo existente en la actualidad es el denominado E-Cash gestionado por la empresa Digi-Cash, consistente en la emisión de unos pequeños archivos que equivalen a dinero digital. Para usar el E-Cash tanto el comprador como el vendedor necesitan tener abierta una cuenta en uno de los bancos que emiten el dinero electrónico, siendo la propia institución financiera la que facilita el software de emisión del dinero efectivo electrónico.

En el momento de generar el billete, el usuario le asigna un valor nominal y un número de serie (el software está programado para generar números de serie aleatorios suficientemente largos para evitar la duplicidad en la generación de los billetes), lo firma y lo envía a la institución financiera quien luego de verificar el número de la cuenta del usuario y la disponibilidad de fondos, procede a firmar el billete con una clave, certificando el valor nominal solicitado, y se lo envía al usuario. Una vez que el usuario ha recibido el billete firmado por el banco, lo puede almacenar en una billetera electrónica o lo puede usar inmediatamente para pagar sus compras en los establecimientos adheridos al sistema. El comerciante, al recibir el billete, lo envía a la entidad emisora y ésta, una vez que ha comprobado la autenticidad de la moneda, acredita el valor del billete en la cuenta del comerciante. Para evitar que el billete sea cobrado dos veces, el banco emisor debe contar con una base de datos que le permita verificar los números de serie de los billetes que han sido pagados.

El problema que se presenta con este procedimiento es que no garantiza en forma absoluta el anonimato del billete pues el banco siempre sabrá la serie de billete que ha firmado y a quién se lo ha firmado, pudiendo conocer, gracias a estos datos, cuándo, cómo y dónde fue gastado el billete. Para solucionar esta situación se ha ideado el sistema de la "firma digital a ciegas", mediante el cual el usuario, antes de enviar la moneda para que la firme el banco, multiplica el número de serie por un factor conocido como "factor ciego" con la finalidad de ocultar el verdadero número de serie del billete electrónico, siendo imposible relacionar el número de serie del billete con el número obtenido después de aplicar el factor ya que sólo el usuario conoce el factor. Cuando el usuario recibe el billete divide el número firmado por el banco aplicando el mismo coeficiente que utilizó para generarlo, obteniendo el número de serie oculto, de manera que es imposible conocer quien ha gastado el billete ya que el banco cuando firmó el billete sólo sabía que se trataba de un billete de un determinado valor solicitado por uno de sus clientes. 


\subsection{El reembolso del dinero}

Con el objeto de fomentar la confianza en el uso del dinero generado a través de medios electrónicos, la Directiva establece y regula el derecho del portador de solicitar el reembolso del dinero electrónico a su valor nominal, en monedas y billetes de banco o por transferencia a una cuenta, sin otros gastos que aquéllos que resulten estrictamente necesarios para ejecutar la operación.

\section{LA ADMISIÓN LEGAL DEL PAGO EFECTUADO}

\section{POR MEDIOS ELECTRÓNICOS}

Una de las principales inquietudes que manifiestan los usuarios de Internet a la hora de efectuar un pago con dinero electrónico se relaciona directamente con su incertidumbre acerca de la admisión legal del pago efectuado a través de estos nuevos medios.

Podemos declarar la admisibilidad legal del pago con dinero efectivo electrónico basándonos en las siguientes premisas:

3.1. La autonomía de la voluntad y la libertad de pacto: según estos principios de carácter general, el pago por medios electrónicos será válido siempre que las partes hayan convenido en ello. En el caso de un pago distinto al dinero efectivo tradicional siempre se requerirá un pacto de esta naturaleza, en virtud de que no puede obligarse al acreedor a aceptar un medio de pago distinto del dinero efectivo.

La validez del contrato electrónico: hoy en día, la mayoría de las legislaciones admiten la validez del contrato efectuado por medios electrónicos. Siendo el pago un medio de ejecución del contrato, en este caso un medio electrónico de ejecución de un contrato electrónico, no vemos objeción alguna para declararlo legalmente admisible.

3.3.

La representatividad del medio de pago: para validar el pago a través de signos representativos del dinero (letras de cambio y cheques), la mayoría de las legislaciones admiten el principio de que el pago puede hacerse en dinero efectivo o en un signo que lo represente, como ha quedado de manifiesto a lo largo de esta ponencia, el dinero electrónico no es más que una representación binaria del dinero tradicional, siendo en todo caso susceptible de conversión a dinero real en beneficio de los derechos del portador. 
